\title{
ESTUDO DA VALIDADE DE UM QUESTIONÁRIO DE AVALIAÇÃO DO DESEMPENHO DO PROFESSOR DE FÍSICA GERAL PELO ALUNO
}

Fernando Lang da Silveira ${ }^{1}$ Marco António Moreira ${ }^{2}$

RESUMO: Este trabalho apresenta um questionário com itens do tipo Likert, o qual que tem o objetivo precípuo de avaliar o desempenho do professor de Física Geral pelo aluno. $\mathrm{O}$ estudo da estrutura interna $\mathrm{e}$ as evidências de validade do questionário relatadas tiveram por base a resposta de 828 alunos, em 40 turmas, sobre 23 professores do Instituto de Física da UFRGS. Versões reduzidas do questionário também são apresentadas.

ABSTRACT: A questionnaire constructed with Likert type items and specifically designed to evaluate teacher's performance by the student in introductory college physics is presented. The internai structure study and the validity evidences reported in the paper are based on answers provided by 828 students, from 40 different sections, regarding 23 college physics of the Institute of Physics of thè Federal University of Rio Grande do Sul. Short versions of questionnaire are aiso presented.

\section{Introdução}

A avaliação do professor pelo aluno, apesar de ser vista com reservas por muitos professores, pode, na prática, não ter nada de ameaçadora e ser utilizada como um instrumento útil para a melhoria do ensino universitário. Moreira (1981) discutiu, extensamente, a questão da avaliação do professor pelo aluno como um mecanismo de realimentação capaz de contribuir para a melhoria do ensino superior. Silveira e Moreira (1984), Silveira, Moreira e Nunes (1985), Silveira (1989) e Pinent, Silveira e Moraes (1993) apresentaram instrumentos e relataram resultados da aplicação dos mesmos, obtendo evidências de validade para esse tipo de avaliação na Universidade Federal do Rio Grande do Sul, Pontifícia Universidade Católica do Rio Grande do Sul e Universidade do Vale do Rio dos Sinos. Os questionários, apresentados nesses estudos, não eram específicos para um particular tipo de professor e/ou disciplina, tendo sido utilizados em disciplinas tão diferentes como as de Economia, Arquitetura, Matemática, Física, etc., atingindo um total de mais de uma centena e meia de professores, quase quatro centenas de turmas e cerca de oito mil alunos.

Neste trabalho, apresentamos um questionário que foi idealizado para avaliar o desempenho de professores de Física Geral. O Instituto de Física da Universidade Federal do Rio Grande do Sul oferece, entre outras disciplinas, quatro de Física Geral que são freqüentadas, principalmente, por alunos das Engenharias. Essas disciplinas possuem aulas teóricas e de laboratório. O instrumento, Questionário de Avaliação do Desempenho do Professor - Modelo C - Disciplinas de Física Geral (vide Apêndice), destina-se a avaliar o desempenho de professores por seus alunos em disciplinas com tais características. 


\section{O Instrumento}

O questionário é constituído por 46 itens do tipo Likert (Likert, 1976): afirmações com as quais o aluno deve expressar o seu grau de concordância ou discordância em uma escala de 5 pontos. Os primeiros 38 itens referem-se ao desempenho do professor na opinião do aluno; os 8 itens adicionais permitem ao aluno manifestar sua opinião sobre diversos aspectos, tais como: se a disciplina é importante para a sua carreira, se gosta de Física, se assistiu à maior parte das aulas, se dispendeu muito esforço nessa disciplina, etc. Como veremos mais adiante, esses itens adicionais também são importantes para a discussão da validade da avaliação do professor pelo aluno.

\section{Quantificação das Respostas de um Aluno a um Item}

O aluno, ao responder a qualquer item do questionário, expressa o seu grau de concordância ou discordância com a afirmação feita em uma escala de cinco pontos: Concordo plenamente (CP), Concordo (C), Indeciso ou não tenho opinião (NO), Discordo (D), Discordo totalmente (DT).

Os itens que expressam um conteúdo favorável ao professor têm as respostas quantificadas da seguinte forma: CP -> escore 5, C -> escore 4, NO -> escore 3, D -> escore 2, DT -> escore 1. Os itens que expressam um conteúdo desfavorável ao professor (vide, nas tabelas 1 e 2, os itens indexados com asterisco (*)) têm as respostas quantificadas da seguinte forma: DT $\rightarrow$ escore 5, D -> escore 4, NO -> escore 3, C -> escore 2, CP -> escore 1. Dessa forma, o escore 5 sempre está associado a uma opinião positiva do aluno em relação ao seu professor, isto é, à concordância plena com item de conteúdo favorável e discordância total com item de conteúdo desfavorável, e o escore 1 sempre está associado a uma opinião negativa em relação ao seu professor.

\section{Quantificação dos Escores Totais}

Os itens do questionário que se referem ao professor (38 itens iniciais) podem ser classificados em dois grandes grupos: o primeiro deles contém 30 itens relativos ao seu desempenho em aulas teóricas (vide Tabela 1); o segundo contém 8 itens relativos ao seu desempenho em aulas de laboratório (vide Tabela 2). Assim sendo, produzem-se dois escores totais (somatório dos escores nos itens respectivos), denominados TEORIA e LABORATÓRIO. O somatório dos escores em todos os 38 itens produz um escore total - denominado GERAL - que quantifica a opinião geral do aluno sobre o seu professor. Essa forma de construir os três totais, coerente com a proposta de Likert (1976), leva a escores com valor mínimo igual ao número de itens e com valor máximo igual ao número de itens vezes cinco. O ponto neutro da escala situa-se no escore igual ao número de itens vezes 3 . Outra possibilidade é a de dividir cada um dos escores totais pelo respectivo número de itens que os constituem, obtendo-se três médias compreendidas no intervalo fechado 1 a 5 .

O Departamento de Física do IF-UFRGS oferece aos professores interessados, próximo ao término do semestre letivo, os questionários e as grades de respostas. O professor retorna ao Departamento as grades preenchidas para o processamento. Posteriormente, ao professor é fornecido um relatório com os resultados de seus alunos o qual informará: 1- Para cada item do questionário a distribuição de frequiências das respostas através das cinco alternativas, a média e o desvio-padrão. 2- O histograma, a média e o desviopadrão dos três escores totais (TEORIA, LABORATÓRIO e GERAL). 3- As 
manifestações por escrito dos alunos na grade de respostas. Todas essas informações, além de serem comunicadas

ao professor avaliado, ficam arquivadas no Departamento. Adicionalmente, podem ser consultadas por qualquer interessado em um documento disponível na biblioteca do IFUFRGS.

Na próxima seção, apresentamos a discussão da estrutura interna (análise fatorial e coeficientes de fidedignidade) do questionário. Todas as análises estatísticas apresentadas nas seções 5 a 8 foram produzidas com o auxílio do software "SPSS for Windows -Release 6.0" (Norusis, 1993).

\section{A Estrutura Interna do Questionário}

O estudo dá estrutura interna do questionário foi realizado sobre as respostas de 828 alunos, em 40 turmas, as quais cursaram disciplinas ministradas por 23 professores do Instituto de Física da UFRGS em 1995 e 1996. Desses 23 professores, 11 foram avaliados em mais de uma turma como se verificará na seção 6 . O total de alunos respondentes era 896, sendo as respostas de 68 alunos eliminadas da análise por um dos seguintes critérios: 1-Frequiência de respostas na alternativa NO (indeciso ou não tenho opinião) ou sem resposta maior do que 15 itens. 2- Resposta NO, ou D, ou DT (indeciso, ou discordo, ou discordo totalmente) no item 46 (Respondi ao questionário com atenção.).

Analisando-se o conteúdo dos itens que se referem ao professor (itens 1 a 38), destacam-se dois grupos de itens: o primeiro deles, denominado TEORIA, é constituído por 30 itens (vide Tabela 1) e versa sobre o desempenho do professor em aulas teóricas; o segundo, denominado LABORATÓRIO, é constituído por 8 itens (vide a Tabela 2) e versa sobre o desempenho do professor em aulas de laboratório. Esses dois grupos de itens foram submetidos a uma análise fatorial através do "método dos grupos múltiplos de Thurstone" (Nunnally, 1978; Wherry, 1984). Esse método, contrariamente a outros métodos de análise fatorial nos quais os fatores aparecem a posteriori (Mulaik, 1972), parte da postulação a priori de fatores em grupos de variáveis (itens), testando empiricamente corroborando ou não a inclusão de cada item no fator postulado. O método também permite verificar se algum item está mal classificado (pertence a outro fator diferente do postulado ou a nenhum). Cada fator é uma combinação linear das variáveis (em geral, o somatório com igual peso) previamente de signadas. Em seguida, para cada variável é calculado o coeficiente de correlação desta com cada um dos fatores postulados (carga fatorial). O "método dos grupos múltiplos de Thurstone", contrariamente aos métodos que produzem fatores inicialmente ortogonais, gera-os relacionados, sendo os coeficientes de correlação entre cada par de fatores calculado a posteriori.

Nas tabelas 1 e 2, encontram-se a média e o desvio-padrão do escore em cada item bem como a carga fatorial nos fatores 1 (TEORIA) e II (LABORATÓRIO).

\begin{tabular}{|c|c|c|c|c|}
\hline. & 42 & 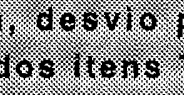 & 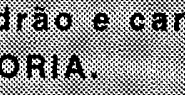 & as \\
\hline ITEM & MÉDIA & DESVIO PADRÃO & FATORI (TEORIA) & $\begin{array}{c}\text { FATORII } \\
\text { (LABORATÓRIO) }\end{array}$ \\
\hline 01 & 4,54 & 0,69 & 0,62 & 0,46 \\
\hline $02 *$ & 3,75 & 1,14 & 0,74 & 0,51 \\
\hline $03 *$ & 3,85 & 1,12 & 0,72 & 0,51 \\
\hline 04 & 4,10 & 0,91 & 0,59 & 0,49 \\
\hline $05^{*}$ & 4,01 & 0,99 & 0,42 & 0,29 \\
\hline 06 & 4,11 & 0,94 & 0,47 & 0,28 \\
\hline 07 & 3,87 & 1,09 & 0,69 & 0,45 \\
\hline
\end{tabular}




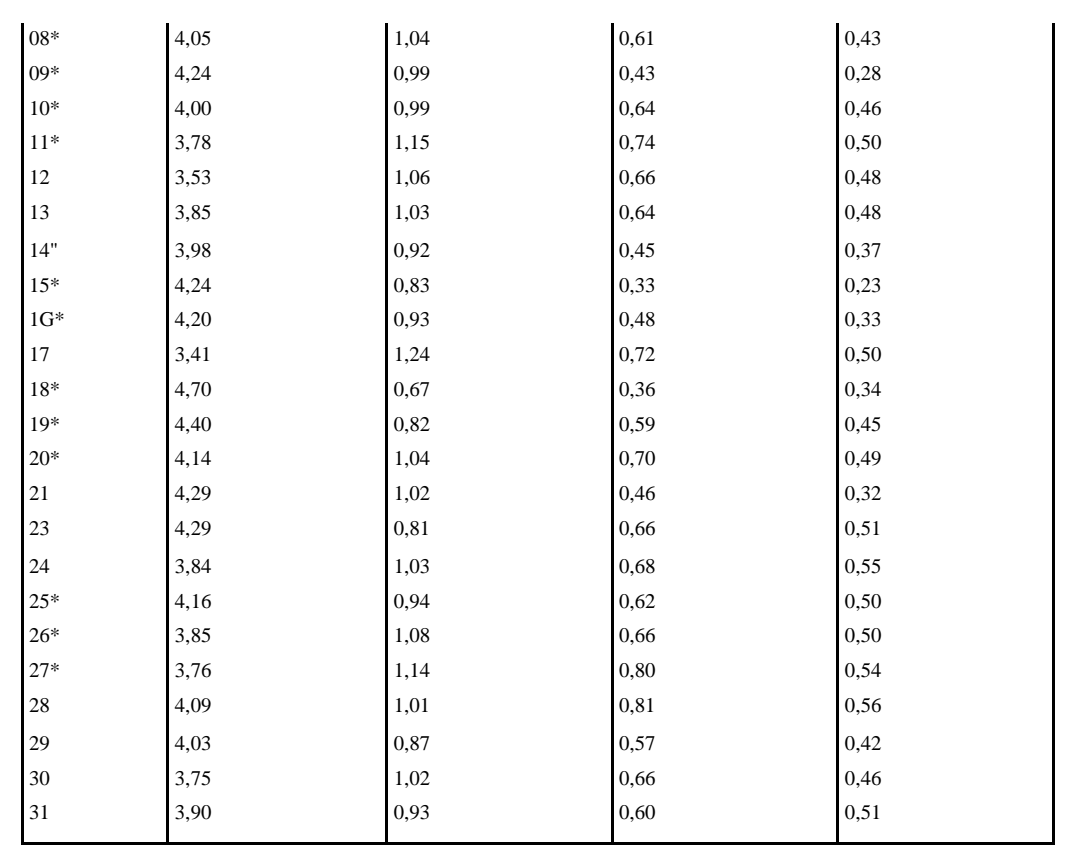

item que expressa uma opinião desfavorável ao professor.

\begin{tabular}{|c|c|c|c|c|}
\hline ITEM & MÉDIA & DESVIO PADRÃO & FATO R I (TEORIA) & $\begin{array}{c}\text { FATORII } \\
\text { (LABORATÓRIO) }\end{array}$ \\
\hline 22 & 4,16 & 0,90 & 0,58 & 0,67 \\
\hline 32 & 3,95 & 0,95 & 0,50 & 0,74 \\
\hline 33 & у 4,29 & 0,80 & 0,49 & 0,72 \\
\hline $34 *$ & 4,10 & 0,95 & 0,41 & 0,68 \\
\hline 35 & 3,86 & 0,93 & 0,54 & 0,67 \\
\hline 36 & 4,12 & 0,83 & 0,59 & 0,74 \\
\hline $37 *$ & 4,08 & 0,90 & 0,42 & 0,63 \\
\hline $38 *$ & 4,10 & 0,88 & 0,51 & 0,70 \\
\hline
\end{tabular}

* - item que expressa uma opinião desfavorável ao professor.

Todas as cargas fatoriais são positivas e estatisticamente significativas em nível inferior a $1 \%$, sendo seus valores superiores a 0,30 no fator postulado. As diferenças, para cada item, entre as cargas nos fatores 1 e II (testadas conforme Guilford e Fruchter (1973)), são estatisticamente significativas em nível inferior a $1 \%$ com uma única exceção apenas: item 18 .

Dessa forma, fica corroborada a inclusão de todos os itens no respectivo fator postulado; inclusive o item 18, pois ele tem carga fatorial no fator $1 \mathrm{um}$ pouco maior do que no fator II. A existência, em todos os itens, de carga fatorial positiva no outro fator - às vezes quase igual àquela do fator postulado - evidencia a não-ortogonalidade dos dois fatores. O coeficiente de correlação entre os dois fatores é 0,73 , implicando a possibilidade de se construir também um escore total nos 38 itens: esse escore, denominado GERAL, quantifica a opinião global do aluno a respeito do seu professor.

Na Tabela 3, encontram-se a média, o desvio-padrão dos três escores totais, bem como uma estimativa do coeficiente de fidedignidade de cada total: o coeficiente alfa (Cronbach, 1951). Os elevados coeficientes de fidedignidade corroboram, empiricamente, a construção desses escores totais. 


\begin{tabular}{|c|c|c|c|}
\hline \multicolumn{4}{|c|}{ 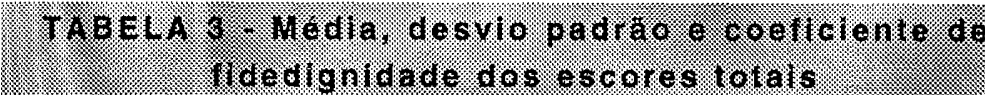 } \\
\hline ESCORE & MÉDIA & PESVIO & FOEFICIENTE DE \\
\hline $\begin{array}{l}\text { TEORIA } \\
\text { LABORATÓRIO } \\
\text { GERAL }\end{array}$ & $\begin{array}{l}120,73 \\
32,66 \\
153,39 \\
\end{array}$ & \begin{tabular}{|l}
18,04 \\
4,96 \\
21,91
\end{tabular} & $\begin{array}{l}0,94 \\
0,85 \\
0,95\end{array}$ \\
\hline
\end{tabular}

\section{Evidências de Validade do Questionário}

Além da usual validade de conteúdo ${ }^{1}$ para os itens do questionário, há a opinião dos alunos sobre a validade do questionário expressa no item 43 (Acho este tipo de questionário válido): 84,7\% (701 alunos em 828) concordaram com a afirmação e apenas 9\% (62 alunos em 828) discordaram; o escore médio nesse item foi alto -4,22 -, indicando a concordância dos alunos com a validade do questionário.

Pesquisou-se, também, a existência de relação entre o escore GERAL (medida global da opinião do aluno sobre o seu professor) e as respostas que os alunos deram aos 8 itens adicionais (vide questionário em anexo). A Tabela 4 apresenta esses resultados.

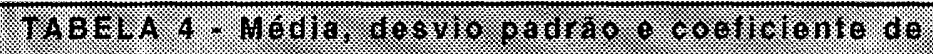

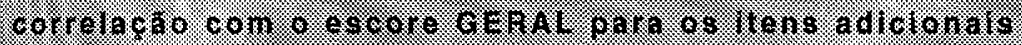

\begin{tabular}{|c|c|c|c|}
\hline ITENS ADICIONAIS & Média & Desvio padrão & $\begin{array}{l}\text { Coef. } \\
\text { correlação }\end{array}$ \\
\hline 39 - Considero que fui bom aluno desta disciplina. & 3,75 & 1,01 & 0,20 \\
\hline 40 - Acredito que esta disciplina é importante para minha carreira. & 3,69 & 1,19 & 0,23 \\
\hline 41 - Dediquei bastante esforço ao estudo desta disciplina. & 3,46 & 1,15 & 0,13 \\
\hline 42 - Assisti a maior parte das auías desta disciplina. & 4,35 & 0,90 & 0,16 \\
\hline 43 - Acho este tipo de questionário válido. & 4.22 & 0.94 & 0,09 \\
\hline 44 - Tenho a impressão de que aprendi bastante nesta disciplina. & 3,85 & 1,03 & 0,47 \\
\hline 45 - Gosto de Física. & 3,86 & 1,15 & 0,19 \\
\hline 46 - Respondi o questionário com atenção. & 4,68 & 0,47 & 0,21 \\
\hline
\end{tabular}

Conforme se constata na Tabela 4, com exceção do item 44, os coeficientes de correlação entre o escore GERAL e os escores nos itens adicionais são baixos (iguais ou inferiores a 0,23 ), ou seja, a opinião que o aluno tinha sobre o seu professor era quase independente da importância que ele atribuía: à disciplina para a sua carreira, ao fato de gostar de Física, do fato de achar o questionário válido, ao fato de ter assistido a maior parte das aulas, etc. $\mathrm{O}$ único item que se correlacionou, razoavelmente, com o escore GERAL foi o que expressa o quanto o aluno pensou ter aprendido na disciplina $(0,47$ para o item 44); dessa forma, configurou-se uma tendência no sentido de que quanto mais o aluno acreditava ter aprendido, tanto melhor avaliava o seu professor. A existência, de correlações fracas dos demais itens com o escore GERAL constitui-se em um argumento de validade, demonstrando que a opinião do aluno sobre o seu professor era quase independente das suas opiniões sobre outros aspectos.

Dos 23 professores envolvidos neste estudo, 11 foram avaliados por seus alunos em mais de uma turma, alguns em semestres diferentes. Estudamos, então, a capacidade do questionário de discriminar entre os diferentes professores e, até que ponto, foram estáveis as avaliações de um mesmo professor em diferentes turmas. Esperávamos encontrar diferenças marcantes entre os diversos professores e pouca ou nenhuma diferença para um mesmo professor. A tabela 5 apresenta os resultados da análise da 
variância univariada (Winer, 1971) para o escore total GERAL. Na primeira linha, está a análise efetuada com todas as 40 turmas, portanto, contendo as diferenças entre os vários professores. Em seguida, aparecem as análises para cada um dos professores que foram avaliados em diversas turmas, alguns em semestres diferentes. Além da razão F, que permite testar a significância estatística das diferenças nas médias do escore GERAL entre as turmas, são apresentadas a maior e a menor média do escore GERAL por turma e a percentagem da variância explicada (percentagem da variância do escore Geral que é devida às diferenças entre turmas). Esses três valores dão informações sobre a heterogeneidade do escore GERAL através das turmas.

Conforme exposto anteriormente, o escore GERAL pode assumir valores compreendidos entre 38 e 190. Na Tabela 5, observa-se que, em 5 dos 11 professores avaliados em diferentes turmas, apareceram diferenças estatisticamente significativas em nível

\begin{tabular}{|c|c|c|c|c|}
\hline GRUPOS & $\begin{array}{l}\text { MAIOR TOTAL } \\
\text { MÉDIO }\end{array}$ & $\begin{array}{l}\text { MENOR TOTAL } \\
\text { MÉDIO }\end{array}$ & RAZÀOF & $\begin{array}{l}\text { PERCENTAGEM } \\
\text { DA VARIÂNCIA } \\
\text { EXPLICADA }\end{array}$ \\
\hline $\operatorname{TODOS}(n=40)$ & 177,46 & 97,28 & $13,02^{*}$ & $\begin{array}{l}39,2 \\
\text { lliilll }\end{array}$ \\
\hline Professor $1(n=4)$ & 160,78 & 141,89 & $4,44 *$ & 12,2 \\
\hline Professor $2(\mathrm{n}=3)$ & 171,21 & 164,54 & 1,38 & 2,9 \\
\hline Professor $3(\mathrm{n}=3)$ & 166,07 & 154,91 & $4,97 *$ & 9,5 \\
\hline Professor $4(n=: 3)$ & 156,44 & 148,70 & 1,04 & 2,6 \\
\hline Professor $5(\mathrm{n}=3)$ & 172,07 & 153,41 & $8,04 *$ & 20,3 \\
\hline Professor $6(n=3)$ & 151,39 & 132,19 & $6,52 *$ & 17,1 \\
\hline Professor $7(\mathrm{n}=2)$ & 162,20 & 150,20 & 1,23 & 13,3 \\
\hline Professor $8(\mathrm{n}=2) y$ & 165,78 & 160,90 & 0,81 & 2,3 \\
\hline Professor $9(\mathrm{n}=2)$ & 163,20 & 160,57 & 0,25 & 0,1 \\
\hline Professor $10(n=2)$ & 146,13 & 137,62 & 2,53 & 6,2 \\
\hline Professor $11(\mathrm{n}=2)$ & 159,67 & 142,67 & $5,36^{*}$ & 13,6 \\
\hline & \multicolumn{4}{|c|}{ Média para os 11 professores } \\
\hline
\end{tabular}

* - Estatisticamente significativo em nível inferior a 5\% n - Número de turmas.

inferior a 5\%; entretanto, em qualquer desses casos, a diferença entre a maior e a menor média nunca chegou a 20 pontos. A percentagem média da variância explicada do escore GERAL para os 11 professores é apenas 9,7\%. Fica assim configurada uma razoável convergência na avaliação que os alunos de diferentes turmas fazem sobre o mesmo professor, ou seja, o mesmo professor, quando avaliado em diferentes turmas, obteve resultados semelhantes.

Quando foram comparadas as 40 turmas (primeira linha da Tabela 5), ocorreram importantes diferenças que representam até 80 pontos no escore GERAL médio (diferença entre a maior e a menor média), perfazendo cerca de $40 \%$ da variância explicada. Essas diferenças reforçam que o escore GERAL é fidedigno pois, "sem fidedignidade não é possível verificar essas diferenças" (Silveira, 1980, 706). Demonstram, também, a capacidade do escore GERAL de discriminar os diferentes professores.

Todos esses resultados constituem-se em argumentos de validade de construto (Silveira, 1993) para o questionário. 


\section{Características do Professor que Levam o}

\section{Aluno a Recomendá-lo como Bom Professor}

No item 28, o aluno manifestou-se no sentido de recomendar ou não recomendar seu professor como um bom professor. Estudou-se a relação dessa resposta com todas as outras características do professor, abrangidas nos demais itens do questionário. Essa análise teve como objetivo explicitar aquilo que os alunos consideravam como relevante para recomendar ou não um professor.

Procedeu-se a uma regressão múltipla do escore no item 28 contra os demais itens. Na regressão múltipla, a variável dependente (aqui, o escore no item 28) é expressa como uma combinação linear das variáveis independentes (aqui, os demais escores nos itens do questionário) e os pesos com os quais as variáveis independentes entram na combinação linear (equação de regressão) são calculados de modo a maximizar o coeficiente de correlação linear da variável dependente com a combinação linear das independentes (Wherry, 1984). A Tabela 6 apresenta o coeficiente de correlação simples e o coeficiente de regressão (regressor) padronizado de cada um dos itens que permaneceram na equação de regressão; somente nove itens foram retidos, sendo os demais descartados por um procedimento "stepwise". Esse procedimento seleciona, dentre as variáveis independentes, aquelas que significativamente contribuem para o poder explicativo da equação de regressão (Afifi e Clark, 1996), quantificado no coeficiente de correlação múltipla. Todos os coeficientes da Tabela 6 são estatisticamente significativos em nível inferior a $5 \%$.

O regressor padronizado de uma variável (item) é o peso com a qual ela entra na equação de regressão, quando a variável dependente e as independentes são padronizadas $^{2}$. Através da comparação desses coeficientes, pode-se inferir sobre a importância relativa de cada variável na explicação da variável dependente. Dessa forma, os itens 02 e 11 foram os mais importantes para a indicação feita pelo aluno. Como esses itens têm conteúdo negativo e a concordância plena está associada ao escore mais baixo (escore 1), o aluno tenderá a indicar como bom professor aquele que, primordialmente, "dá explicações claras" e "tem didática". Os itens estão listados na Tabela 6, em ordem decrescente do seu peso (regressor padronizado - (3), permitindo, facilmente, a visualização da ordem de importância das características principais do bom professor na opinião do aluno. O coeficiente de correlação múltipla 0,79 indica que os nove itens em conjunto apresentaram poder razoável de explicar o que significou ser bom professor.

\section{Versões Reduzidas do Questionário}

O questionário, na sua versão integral, é constituído por 46 itens, dos quais 38 referemse ao professor. Na Tabela 7, são apresentadas duas versões reduzidas do questionário, uma com 19 itens e a outra com apenas 10 itens. A seleção dos itens de cada versão teve como critério a maximização do coeficiente de fidedignidade do escore GERAL para 19 itens e para 10 itens.

Observa-se que a versão com 19 itens, a metade dos itens da versão integral, apresentou coeficiente de fidedignidade superior a 0,90 e que, mesmo na outra, com apenas 10 itens, ele foi alto. O coeficiente de correlação do escore GERAL, na versão completa com o escore GERAL das versões reduzidas é próximo da unidade ${ }^{3}$. Dessa forma, se o objetivo for o de fazer uma medida global do desempenho do professor por seus alunos, são suficientes muito menos itens do que os que integram a versão completa. 
ITEM

11 - Tem pouca didática

$$
\begin{aligned}
& 02 \text { - Dá explicações pouco claras } \quad 0,64 \\
& 0,18 \\
& 0,62 \quad 0,16
\end{aligned}
$$

$$
01 \text { - Parece dominar a matéria } 0,55 \quad 0,13
$$$$
24 \text { - Propõe problemas interessantes } \quad 0,54
$$$$
0,12
$$

08 - Mostra-se inseguro ao responder perguntas

$$
0,53 \quad 0,12
$$

19 - Parece não planejar as aulas $\quad 0,48 \quad 0,11$

17 - Mantém o aluno atento durante as aulas $\quad 0,58 \quad 0,11$

29 - Aceita o ponto de vista do aluno $\quad 0,46 \quad 0,10$

10 - Parece não se preocupar se o aluno está aprendendo $\quad 0,49 \quad 0,10$

$$
\mathbf{R}=\mathbf{0 , 7 9}
$$

$\mathrm{r}$ - Coeficiente de correlação do escore no item com o item 28.

P - Coef. de regressão padronizado do escore no item contra o escore no item 28.

$\mathrm{R}$ - Coef. de correlação múltipla do escore no item 28 contra todos os demais itens.

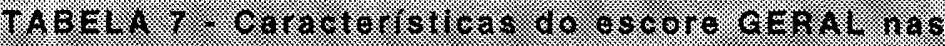

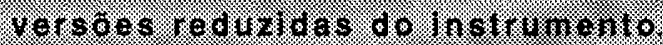

$\begin{array}{llll}\text { ITENS CONSTITUINTES DA VERSÃO } & \text { COEFICIENTE DE } & \text { COEFICIENTE DE CORRELAÇÃO } \\ \text { REDUZIDA } & \text { FIDEDIGNIDADE } & \text { COM O ESCORE GERAL NA } \\ & & \text { VERSÃO COMPLETA }\end{array}$

$\begin{aligned} & 19 \text { itens: } 02,04,06,07,08,10,13,14.17,19, \\ & 21,23,26,27,28,32,33,36,37\end{aligned}$
$\begin{array}{lll} & & \\ 10 \text { itens: } 02,10,13,17,19,27,28,32,33, & 0,88 & 0,97\end{array}$
36

\section{Conclusão}

Este trabalho teve, como tema, a avaliação do desempenho do professor de Física Geral pelo aluno, focalizando, especificamente, a apresentação de evidências de validade de um instrumento que pode ser utilizado para essa finalidade.

Além de se demonstrar que o instrumento completo teve alta fidedignidade e apresentou evidências de validade, contruíram-se formas reduzidas do questionário com cerca da metade e com cerca de um quarto dos itens da versão completa - também confiáveis.

Ainda fez parte deste trabalho um estudo para identificar as características que se relacionavam com o fato de o aluno recomendar o professor como bom. Tal recomendação está mais fortemente relacionada, em ordem decrescente de importância, ao professor dar explicações claras, ter didática, dominar a matéria, propor problemas interessantes, mostrar-se seguro ao responder ao aluno, planejar as aulas, manter o aluno atento, aceitar o ponto de vista deste e preocupar-se com o aprendizado do mesmo.

O questionário, aqui apresentado, pode ser facilmente modificado para avaliar professores em outras disciplinas. No momento em que a universidade brasileira começa a se preocupar com a avaliação do ensino, a testagem de instrumentos, como o que apresentamos, torna-se cada vez mais importante. 


\section{Apêndice}

\section{Questionário de Avaliação do Desempenho do Professor - Modelo C - Disciplinas de Física Geral ${ }^{4}$}

(Leia, com atenção, antes de responder ao questionário)

O objetivo deste questionário é o de colher a opinião do aluno sobre o desempenho do professor. Com isso, o professor terá elementos adicionais para analisar, criticamente, seu desempenho, procurar corrigir eventuais falhas e melhorar o ensino.

Não assine o questionário. Expresse sua opinião livremente. Em hipótese alguma, os resultados do questionário terão influência no seu conceito final. Procure ser imparcial. Na medida em que suas respostas visarem somente a agradar ou desagradar ao professor, terão sido inúteis para ele e seu tempo, perdido.

Nas folhas que seguem você, encontrará várias afirmativas que, de um modo geral, refletem possíveis características ou comportamentos de um professor. Algumas dessas afirmativas são favoráveis e outras, desfavoráveis. Ao lado de cada uma, existe uma escala na qual você deverá assinalar com um X a alternativa que melhor expressa sua opinião sobre ela. $\mathrm{O}$ código é o seguinte:

$\mathbf{C P} \rightarrow$ Concordo plenamente

$\mathrm{C} \rightarrow$ Concordo

NO > Não tenho opinião ou estou indeciso

$\mathrm{D}>$ Discordo

DT $\rightarrow$ Discordo totalmente

Sempre que possível, evite usar a alternativa NO.

Leia, com atenção, cada afirmativa antes de expressar sua opinião.

Se tiver algum comentário adicional, utilize o verso da folha de respostas.

\section{O professor desta disciplina}

1. Parece dominar a matéria.

2. Dá explicações pouco claras.

CP C NO D DT

3. Não estimula o interesse pela matéria. CP C NO D DT

4. Destaca aspectos importantes da matéria. CP C NO D DT

5. Exige pouco raciocínio do aluno. CP C NO D DT

6. É justo na atribuição de notas ou conceitos. CP C NO D DT

7. Procura facilitar a compreensão do aluno. CP C NO D DT

8. Mostra-se inseguro ao responder perguntas

dos alunos.

CP C NO D DT

9. Elabora provas incoerentes com o

que é dado em aula.

CP C NO D DT

10. Parece não se preocupar se o aluno

está aprendendo ou não.

11. Tem pouca didática. CP C NO D DT

12. Estimula soluções criativas para

os problemas propostos.

CP C NO D DT

13 Apresenta a Física como uma

ciência viva, em construção. $\quad$ CP C NO D DT

14. Dá pouca atenção à parte conceituai

da matéria.

CP C NO D DT

15. Parece utilizar livros de texto

desatualizados.

CP C NO D DT

16. Usa critérios de avaliação pouco claros para os alunos.

CP C NO D DT

17. Mantém o aluno atento durante as aulas. CP C NO D DT

18. Freqüentemente falta às aulas. $\quad \mathrm{CP} C \mathrm{NO} D \mathrm{DT}$

19. Parece não planejar as aulas. CP C NO D DT

20. Dá aulas sem entusiasmo. CP C NO D DT

21. Respeita os horários de aula. CP C NO D DT 
22. Estabelece relações entre teoria e experimentação.

CP C NO D DT

23. Parece gostar de dar aulas. CP C NO D DT

24. Propõe problemas interessantes. CP C NO D DT

25. Apresenta a matéria desorganizadamente. CP C NO D DT

26. Apenas repete o que está nos livros. CP C NO D DT

27. Suas aulas são, de um modo geral,

desinteressantes.

CP C NO D DT

28. Poderia ser recomendado como

bom professor.

CP C NO D DT

29. Aceita o ponto de vista do aluno. CP C NO D DT

30. Estimula a participação do aluno em aula. CP C NO D DT

31. Dá evidências de se aprimorar

como professor.

CP C NO D DT

32. Estimula o interesse do aluno

pelas aulas de laboratório.

CP C NO D DT

33. Parece dominar o conteúdo dos experimentos propostos nas aulas de laboratório. CP C NO D DT

34. Dá pouca importância às aulas de

laboratório,

CP C NO D DT

35. Dá instruções claras nas aulas de laboratório.

CP C

NO D DT

36. Procura relacionar com as aulas teóricas os experimentos feitos

nas aulas de laboratório.

CP C NO D DT

37. Nas aulas de laboratório, propõe experimentos sem conteúdo didático.

CP C NO D DT

38. Não define os objetivos dos

experimentos feitos em aulas de

laboratório.

CP C NO D DT

\section{Itens adicionais}

39. Considero que eu fui um bom aluno

desta disciplina.

CP C NO D DT

40. Acredito que esta disciplina é

importante para a minha carreira.

41. Dediquei bastante esforço ao estudo

da disciplina.

CP C NO D DT

42. Assisti à maior parte das aulas

desta disciplina.

CP C NO D DT

43. Acho este tipo de questionário válido. CP C NO D DT

44. Tenho a impressão de que aprendi bastante nesta disciplina.

CP C NO D DT

45. Gosto de Física.

CP C NO D DT

46. Respondi ao questionário com atenção. CP C NO D DT

\section{Referências}

AFIFI, A. A.; CLARK, V. (1996) Compuíer-aíded multivariate

anaiysis. London: Chapman \& Hall. CRONBACH, L. J. (1951) Coefficient

alpha and the internai

structures of tests. Psychometrika, Vol.16, pp. 297-334. (1996) Fundamentos

da testagem psicológica. Porto

Alegre: Artes Médicas. GUILFORD, J. P.; FRUCHTER, B, (1973)

Fundamental statisíics in

psychology and educaíion. New York: Mc-Graw-Hili. LIKERT, R, (1976)

Una técnica para medir actitudes. In:

Summers, G. F.(ed.) Medición de actitudes. México: Editorial 
Trilias, pp. 182-191. MOREIRA, M. A. (1981) Avaliação do professor pelo aluno como

instrumento de melhoria do ensino universitário. Educação e

Seleção, São Paulo. Vol. 4, pp. 109-123. MULAIK, S. A. (1972) The

foundations of factor anaiysis. New

York: McGraw-Hill. NORUSIS, M. J. (1993) SPSS for Windows. Release 6.0. Chicago:

SPSS Inc. NUNNALLY, J. C. (1978) Psychometric Theory. New York:

McGraw-Hill. PINENT, C. E. C.; SILVEIRA, F. L.; MORAES, R. (1993)

Avaliação

do professor pelo aluno: questionário e testes de validação. Es-

tudos em Avaliação Educaciona\, São Paulo. Vol. 8 pp. 65-79. SILVEIRA,

F. L. (1980) Fidedignidade das medidas e diferenças

entre grupos em psicologia e educação. São Paulo: Ciência e

Cultura, São Paulo. Vol. 33(5), pp. 704-707.

(1989) Avaliação do

ensino: os enfoques objetivo e

qualitativo. Educação e Seleção, São Paulo. V. 20, pp. 63-77.

Validação de testes de lápis e papel. (1993) In: MOREIRA,

M. A.; SILVEIRA, F. L. Instrumentos de pesquisa em ensino e aprendizagem. Porto Alegre: EDIPUCRS. pp. 67-101. SILVEIRA, F .L., Moreira, M. A. (1984) Avaliação do desempenho

do professor pelo aluno: evidências de validade de um instrumento. Ciência

e Cultura, São Paulo. Vol. 36(3). pp. 466-472. SILVEIRA, F .L., MOREIRA, M. A., NUNES, A. D. (1985)

Avaliação do desempenho do professor pelo aluno: novas evidências de validade de um instrumento. Ciência e Cultura,

São Paulo. Vol. 37(8), pp. 1237-1240. WHERRY, R. J. (1984) Contributions to correia tional analysis.

London: Academic Press. WINER, B. J. (1971) Statistical principies in experimental

\section{Correspondência:}

Prof. Fernando Lang da Silveira e Prof . Marco António Moreira, Instituto de Física da Universidade Federal do Rio Grande do Sul; Av. Bento Gonçalves, 9500 - Caixa Postal "15051-CEP 91501-970; Porto Alegre. RS. Brasil. Correio eletrônico: lang@if.ufrgs.br, moreira@if.ufrgs.br

Submetido em 10/01/98, aceito para publicação em 03/11/98. 
